\title{
Iniciativa Open Courseware. El caso de la Cátedra Tecnologías Informáticas para la Educación, UNED
}

\section{Olga Ligia Brenes Matarrita}

Encargada de Cátedra de Tecnologías Informáticas para la Educación. Programa de Informática Educativa. Escuela de Educación. Universidad Estatal a Distancia; obrenes@uned.ac.cr

Recibido: 10 de mayo de 2011

RESUMEN

El presente trabajo realiza una descripción de la iniciativa de Software Educativo Abierto u Open Course Ware $(\mathrm{OCW})$ en las instituciones de educación superior. Se tratará, entre otros aspectos, la experiencia de la incorporación en el proyecto OCW de dos cursos de la Cátedra Tecnologías Informáticas para la Educación coordinada por la Mag. Olga Ligia Brenes Matarrita, los cuales se imparten dentro del plan de estudios del Programa de Informática Educativa de la Escuela de Ciencias de la Educación, de la Universidad Estatal a Distancia (UNED) de Costa Rica.

\section{PALABRAS CLAVE}

Software Educativo Abierto, Consorcio mundial, libertad de información, formatos digitales, ambientes de aprendizaje, materiales didáctico.
Aprobado: 28 de octubre de 2011

\section{SUMMARY}

This paper provides an overview of the Open Educational Software Initiative or Open CourseWare $(\mathrm{OCW})$ in institutions of higher education. It will inter alia incorporate the experience of the OCW project of two years of the Chair for Information Technology Education Computer Education Program at the School of Educational Sciences of the Universidad Estatal a Distancia (UNED) of Costa Rica.

\section{KEY WORDS}

Open Educational Software, Global Consortium , Freedom of information, digital formats, learning environments, educational material.

\section{INTRODUCCIÓN}

Plasencia (2006) inició a partir de los años go con la incorporación de Internet, todo un movimiento, que ha permitido en la época actual su aprovechamiento en contextos educativos, logrando la posibilidad de publicar vía Web, documentos en formato electrónico que aportan múltiples ventajas a los ambientes de aprendizaje. Lo anterior conlleva la superación de limitaciones que años atrás impedían la divulgación de la información en formatos digitales y por consiguiente, la socialización del conocimiento por los mismos medios.

Este soporte para la publicación le imprime un nuevo concepto de libertad a la información y ayuda a que el conocimiento posea un carácter universal. Este es precisamente el objetivo que persigue Open Course Ware (OCW), que surgió según Universia (2007) como una decisión inicial de 
incorporar electrónicamente un concepto de editorial digital, disponible mediante la web. Su inicio se dio en el año 2001 y sus gestores intelectuales fueron en forma conjunta el Instituto Tecnológico de Massachusetts (MIT), la Fundación William and Flora Hewlett y la Fundación Andrew W. Mellon.

\section{- Open Course Ware}

OCW-Universia (2007) es una red de universidades que forma parte de la iniciativa innovadora llevada a cabo mediante Internet por el MIT. Esta iniciativa permite la accesibilidad de manera libre a todo tipo de materiales educativos ofrecidos por esta institución y dirigida a una población docente mundial.

El propósito inicial del proyecto OCW-Universia (2007) fue facilitar el acceso libre a recursos educativos para docentes, estudiantes y autodidactas a nivel mundial, sin fines lucrativos. Sin embargo, la aceptación de la propuesta por parte del sector académico, potenció un nuevo objetivo, como fue crear un modelo eficiente que otras universidades pudieran utilizar, cuando quisieran publicar sus propios materiales pedagógicos.

\section{METODOLOGÍA}

La metodología llevada a cabo para la creación del presente artículo consistió en una revisión de literatura, para ello se consultaron bases de datos especializadas como: EBSCO, la cual es una base de datos multidisciplinaria que permite el acceso a publicaciones periódicas académicas y científicas; PROOUEST, servicio de información en línea, desde donde se accede a un conjunto de bases de datos especializadas que brinda consulta a publicaciones periódicas tanto en revistas como en periódicos del ámbito académico y científico; Scientific American, revista de divulgación científica, con énfasis en las áreas de ciencia y tecnología; además de revistas electrónicas especializadas en el campo de la tecnología para Educación a Distancia y en el contexto del e-Learning. De todas estas fuentes mencionadas se analizaron artículos y estudios relacionados con el tema, en los idiomas inglés y español.

La revisión de literatura se llevó a cabo en dos fases, utilizando el análisis de contenido como principal técnica. La técnica de análisis de contenido permitió analizar un número significativo de libros y artículos, publicados en la red mundial, sobre el tema planteado.

Una primera fase fue convergente, es decir, se utilizó como discriminador el período de publicación 2010-2011. Los descriptores tomados en cuenta fueron: software educativo abierto, consorcio mundial, libertad de información, recursos educativos en formatos digitales, ambientes de aprendizaje en línea.

Una segunda fase fue divergente, para ello se seleccionaron informes, artículos y documentos donde se comprobara a través de la lectura de los resúmenes y títulos que guardaban estrecha relación con el tema de software educativo abierto.

Se procedió a analizar cada documento y se filtraron utilizando como criterio la relación estrecha con el tema. Luego se realizó el análisis de los documentos filtrados, para identificar ejemplos de buenas prácticas en diferentes instituciones de educación superior sobre el tema de software educativo abierto y descartar los que no se concentraran en este tópico.

\section{Surgimiento de Open CourseWare}

De acuerdo al MIT-OCW (2002), la iniciativa OCW parte, en un principio, como una red que contiene publicaciones de contenido de todos los cursos del MIT, en forma libre, gratuita y a nivel mundial, de manera permanente. Por lo tanto, el instituto habilita en ese espacio todos los cursos contenidos en su oferta académica, 
abarcando tanto los niveles de pregrado como de postgrado.

Es importante destacar que no todos los contenidos y materiales didácticos se ofrecen en forma completa, en algunos casos solo se presenta una parte de ellos, esto porque los materiales únicamente se ofrecen como apoyo a los procesos de aprendizaje.

Según MIT-OCW (2002), los usuarios que desean cursar las materias que ofrece OCW tienen acceso a los documentos clasificados en tres categorías: a) planificación de cursos, los cuales contienen los programas de cada curso, temarios, objetivos pedagógicos y calendarios de actividades entre otros; b) contenidos, dentro de esta categoría se ubican la bibliografía, material audiovisual y material auxiliar; c) actividades pedagógicas, que contienen las asignaciones o tareas, los ejercicios, test, proyectos, prácticas de laboratorio, guías de estudio y materiales de aprendizaje.

En algunos casos se ofrecen partes de pruebas evaluativas, pero el usuario no tiene acceso a exámenes completos, esto en el caso de que las estrategias de evaluación de los aprendizajes contemplen esta modalidad evaluativa. Otro aspecto fundamental de destacar es que los estudiantes de estos cursos no tienen acceso a comunicaciones en línea con ninguno de los profesores, de manera que el aprendizaje es completamente autónomo.

\section{- Aspectos a tomar en cuenta para ingresar a los cursos de la red de OCW}

Entre los aspectos a tomar en cuenta para el ingreso libre de los usuarios a los cursos de la red de OCW, destaca el hecho de que no es necesario inscribirse o registrarse en la página del MIT. El sentido de ello radica en que los usuarios que deciden llevar un curso no obtendrán al finalizar ningún crédito o grado académico. Lo anterior permite que quienes cursan las materias lo puedan hacer libremente atendiendo sus propias necesidades, ritmos e intereses por aprender en forma independiente y autónoma, de manera que el objetivo del MIT es que las personas con interés en su autoformación voluntaria, independiente y permanente lo puedan hacer bajo esta modalidad de aprendizaje libre y gratuito (MIT-OC.OW 2002).

Específicamente el proyecto se dirige a apoyar en las labores docentes a profesores y en el proceso de aprendizaje a los estudiantes, es por lo anterior que ambos agentes del proceso educativo, tienen acceso a la revisión y análisis de materiales didácticos. Y si éstos consideran esos materiales pertinentes, los pueden descargar con facilidad y adaptar a su conveniencia, siempre y cuando se respeten los derechos de propiedad intelectual del MIT. (MIT-OC.OW 2002).

\section{- Historia del MIT OCW}

Graf y Albright (2007) resaltan que la iniciativa del MIT-OCW comenzó como una idea propuesta por el Instituto de Formación Continua de MIT (MIT Lifelong Learning Institute), que pretendía llevar una formación permanente a los graduados de su institución. No obstante, conforme el proyecto se iba desarrollando se iba haciendo cada vez más extenso.

El MIT (2002) realizó en el año 1999, a través de sus facultades, un análisis que pretendía replantearse la manera en que Internet se pudiera utilizar con miras a fortalecer la misión de la institución, de forma que se pudiera lograr un mayor acercamiento al conocimiento y mejorar la educación de sus estudiantes.

En el año 2000, el MIT presenta la propuesta y, en el año 2002, publica su primera versión en su sitio, la cual inició con 50 cursos. Para noviembre del año 2007, completa su primera publicación con la totalidad de su plan de estudios, con más de 1800 cursos y con todas sus disciplinas académicas. El equipo de OCW se propone como práctica constante actualizar 
los contenidos de los cursos que ya existen, así como la implementación de nuevos cursos y servicios en su sitio Web (MIT-OCW 2002).

Charles (2002), rector del MIT, destaca que el instituto ha logrado su objetivo con la propuesta de OpenCourseWare iniciada en el 2001, ya que esta iniciativa se ha constituido en un camino de socialización y difusión del conocimiento, que ha formado un espacio accesible a docentes y estudiantes a nivel mundial, lo cual conllevará a un movimiento potenciador de mejora de la calidad de la enseñanza y de la calidad de vida del ser humano, y que trasciende las fronteras geográficas.

\section{- Planteamientos que propician la incorporación al OCW}

Existen diferentes razones que mueven a las instituciones de educación superior hacia un interés por pertenecer a este proyecto. De acuerdo con Wiley (2010), profesor de Tecnología de la Educación de la Universidad de Utah: "el contenido abierto es una publicidad potentísima para las universidades" (p. 1).

Los fundamentos que respaldan la demanda de las universidades por pertenecer al proyecto son las siguientes: a) acogerse a la plataforma OCW supone un posicionamiento institucional ante un fenómeno de impacto mundial, b) avance del conocimiento que libera recursos didácticos y extiende mecanismos de localización, c) estímulo de la innovación y perfeccionamiento de los recursos docentes utilizados por los profesores, d) acercamiento de los profesores a las TIC, e) oportunidad para abordar el ordenamiento de la propiedad intelectual y el reconocimiento de la autoría, y f) mayor proyección de la misión de la institución educativa (OCW-Universia, 2007, p. 2).

De acuerdo a la Universidad Nacional de Educación a Distancia de España (UNED, 2007), para que el proyecto realizado por una universidad dentro del OCWC obtenga beneficios se deben valorar ciertos aspectos como los siguientes: a) el proyecto debe ser integrado en el Plan Estratégico institucional, b) la flexibilidad y adaptabilidad del proyecto a la institución, c) el compartir ayudas, recursos, experiencias y buenas prácticas, d) la ampliación de la repercusión y alcance del trabajo, y e) las instituciones que forman parte del consorcio refuerzan la imagen de la propia institución (p. 1).

Otro aspecto a tomar en cuenta es que la institución incorporada al proyecto asume como un compromiso la colaboración y promoción de una idea común en el sitio, de manera tal que no exista un desfase con los principios ya existentes y así se mantenga una misma línea en cuanto a lineamientos y diseño del entorno.

Entre las condiciones que se requiere para pertenecer al consorcio OCW, Universia (2007, p. 10) menciona las siguientes:

» Ser una institución debidamente acreditada.

» Publicar los materiales docentes estructurados por asignaturas. El mínimo exigido es de 10 asignaturas.

»Comprometerse en desarrollar propuestas y compartir buenas prácticas que promuevan proyectos similares en calidad, estructura y vocabulario.

» Mantener un OCW site que cumpla las condiciones establecidas.

»Ofrecer y publicar los materiales de forma gratuita y sin fines comerciales.

» Los materiales deben estar limpios en lo referente a la propiedad intelectual.

» Publicar los materiales en el OCW site conlleva autorizar a su uso, reutilización/adaptación, traducción y redistribución a terceros.

„EI OCW site debe ser universalmente accesible vía Internet. 
El acceso a los contenidos de OCW se puede realizar de diferentes maneras, esto es: por áreas temáticas, por autores, por palabras clave o por universidades.

De acuerdo con Universia (2007), todos los materiales que las universidades tengan dispuestos en OCW deben contar con licencia Creative Commons, esto significa que todos esos materiales se ceden a los usuarios para que estos los puedan utilizar, ya sea tal y como se publican, o si lo prefieren los puedan modificar y reutilizar, siempre que no sea con fines comerciales, sino que su uso responda a contextos meramente educativos y sin fines de lucro. Por lo anterior, quien los utiliza debe dar el debido reconocimiento público a la institución propietaria de los materiales.

\section{- Experiencias de instituciones en el Consorcio OCW}

La iniciativa OCW gestada por el MIT, desde su nacimiento hasta la fecha, ha despertado el interés en el ámbito mundial de otras instituciones educativas de prestigio a nivel superior; por ende, cada vez más y más universidades desean pertenecer a este consorcio, que ahora se puede catalogar como de carácter mundial.

Según Universia (2007), son ya más de 130 universidades de todo el mundo las que pertenecen al proyecto OCW y entre todas estas instituciones se suman miles de asignaturas en diferentes idiomas.

Dada la magnitud que ha tomado este proyecto es que se crea en el año 2006 el Consorcio OCW (OCWC), el cual tiene como fin coordinar las acciones a nivel mundial de las distintas universidades y organizaciones pertenecientes a este. Las organizaciones agregadas a este consorcio tienen como fin coordinar y dar soporte a las universidades que las integran: este es el caso de Universia, entidad que se ha comprometido fuertemente con el proyecto y ha establecido vínculos con la Universidad Politécnica de Madrid, promoviendo su desarrollo y el de otras universidades de esta red.

\section{El caso de OCW Universia}

Según Universia (2007): "OCW es un ejemplo entre las iniciativas que en los últimos tiempos han emergido para promover el acceso libre y sin restricciones al conocimiento" (p. 1).

Universia (2007) está formada por un conjunto de universidades españolas, portuguesas y latinoamericanas que han tomado la decisión de unirse a OCWC tomando como base su afinidad tanto cultural como geográfica.

La decisión de Universia (2007) de formar un consorcio de universidades incorporadas al proyecto surge atendiendo a las potencialidades que éste encierra. Es así como en forma voluntaria colabora con el MIT para difundir los materiales que el instituto oferta, realizando las traducciones de los materiales de los cursos y ofreciéndolos en su sitio, de manera que los docentes de habla hispana tengan acceso a estos fácilmente.

Como aspectos logísticos, se pueden mencionar que el OCWC establece para mantener comunicación con sus miembros dos reuniones al año, donde se desarrollan diferentes actividades y foros de discusión, por medio de las cuales se busca establecer acciones que permitan un mejor funcionamiento del proyecto, de modo que entre todos los participantes se definan propuestas tendientes a tomar en cuenta los avances e innovaciones tecnológicas y los requerimientos de formación que existen en la actualidad Universia (2007).

Por otro lado, se propicia que todos los miembros mundiales del consorcio tengan acceso a la totalidad de los sitios que se encuentran en el proyecto OCW. En este sentido, se debe destacar que para el consorcio Universia OCW es fundamental la coordinación que se pueda 
llevar a cabo con todos los integrantes, ya que a pesar de que esta es una iniciativa que promueve la flexibilidad y adaptabilidad a situaciones diversas, las similitudes y homogeneidad de los sitios es elemental para facilitar la utilidad que le puedan dar los usuarios, por ello se establece que todos los sitios deben atender a los mismos principios de diseño.

La modalidad de participación de las universidades en el proyecto puede ser individual o mediante la integración de consorcios llamados, según Universia (2007, p. 6), "capítulos territoriales", como es el caso del Capítulo Iberoamericano OCW-Universia, que está integrado por diferentes universidades españolas, portuguesas y latinoamericanas.

En este caso, el proyecto se inició atendiendo a sus afinidades culturales y geográficas en el contexto iberoamericano, para así asegurarse una mejor representación en el consorcio mundial. Además la estrategia de agruparse en universidades por regiones permite el aseguramiento de la cooperación entre sus miembros para adherir a nuevas universidades.

Universia (2007) expone que la incorporación al consorcio OCW de las universidades, mediante el Capítulo Iberoamericano OCW-Universia, representa una serie de ventajas y mayores oportunidades que si lo hicieran en forma individual.

Actualmente, el Capítulo Iberoamericano OCW-Universia cuenta con 36 universidades entre las que se encuentra la Universidad Estatal a Distancia (UNED) de Costa Rica.

\section{- El caso de la Universidad Estatal a Distancia en Costa Rica en el proyecto OCW}

La Universidad Estatal a Distancia (UNED) es una institución de carácter público que goza de autonomía. Fue creada en el año 1977 como respuesta al aumento en la demanda de la educación superior en el país, a causa de la expansión demográfica y de las aspiraciones de algunos sectores de la población de fortalecerse con la cultura que ofrece la Universidad (UNED, 2005).

La Universidad Estatal a Distancia tiene como misión ofrecer educación superior a todos los sectores de la población, especialmente a aquellos que por razones económicas, sociales, geográficas, culturales, etáreas, de discapacidad o de género, requieren oportunidades para una inserción real y equitativa en la sociedad (UNED, 2005).

Para ello, hace uso de los diversos medios tecnológicos que permiten la interactividad, el aprendizaje independiente y una formación humanista, crítica, creativa y de compromiso con la sociedad y el medio ambiente (UNED, 2005).

Hernández (2001), funcionaria de la UNED de Costa Rica y coordinadora del proyecto en la institución, representó en el año 2009 a este centro de estudios superiores, en la reunión anual del Consorcio OCW, realizada en Monterrey, México.

Esta participación se logró una vez que la Junta Directiva del Consorcio aprobó en el mismo año la solicitud planteada por la UNED, en la que manifestaba su interés por participar en el proyecto.

La participación en este evento responde a la atención de los siguientes objetivos, según lo manifiesta Hernández (2009, p.1): a) presentar los votos de la Universidad, tanto para la elección de los nuevos miembros de la Junta Directiva del Consorcio OCW, como los que correspondían a los estatutos que se proponía cambiar en los reglamentos, b) establecer contacto con los encargados de Creative Commons, c) establecer contacto con los encargados de EduCommons, d) establecer contacto con otros miembros del Consorcio .

Hernández (2001), con respecto al establecimiento de contactos, resalta que la idea primordial de establecer estos contactos es 
determinar las directrices a seguir en el desarrollo del proyecto, referidas específicamente al contexto de la institución. Por otro lado, manifiesta que la importancia de realizar estos contactos radica en que, en estos encuentros, se dictan los lineamientos que permiten a la UNED continuar con el desarrollo del proyecto y realizar las coordinaciones necesarias con los profesionales académicos, que se encargarán del diseño instruccional de los cursos, y con el equipo interdisciplinario que asumirá, entre otras funciones, las de diseño del sitio, aspectos técnicos y administrativos, establecimiento de licencias de los materiales, entre otras actividades.

Una vez que la UNED realiza con el Consorcio OCW todas las coordinaciones correspondientes mediante los responsables del proyecto de la institución, se pone en marcha la oferta abierta de cursos de dos de las escuelas que forman parte de la Vicerrectoría Académica. Actualmente, la UNED cuenta con cuatro cursos en OCW, pertenecientes a las escuelas de Ciencias de la Educación y Ciencias Exactas y Naturales.

\section{- Experiencia en el proyecto OCW de la cátedra Tecnologías Informáticas para la Educación}

La cátedra Tecnologías Informáticas para la Educación, del programa de Informática Educativa, de la Escuela de Ciencias de la Educación, ha incluido dos cursos en el proyecto OCW, luego de llevar a cabo todos los trámites y procesos correspondientes para ello. La inclusión se dio una vez que la coordinadora del proyecto OCW, Diana Hernández Montoya, contactara a la encargada de la cátedra, la Mag Olga Ligia Brenes Matarrita, a quien hizo partícipe de la iniciativa.

Los cursos de la cátedra Tecnologías Informáticas para la Educación que se incorporan al proyecto OCW son: Tecnologías de Aprendizaje
Compartido y Distribuido, que se encuentra en el nivel de Licenciatura de la Carrera de Informática Educativa, y el curso Telecomunicaciones Educativas I, que se encuentra en el nivel de Bachillerato de la misma Carrera.

Ambos cursos se ofertan en línea con un nivel avanzado de virtualidad, es decir, todas las actividades se desarrollan en el aula virtual creada para este fin, los cursos no tienen contemplado dentro de las actividades de evaluación de los aprendizajes los exámenes escritos, las estrategias de evaluación se centran en proyectos prácticos en línea, foros de discusión temática, estudios de caso, portafolios electrónicos, investigaciones temáticas mediante trabajo colaborativo en línea, análisis de software libre para la educación y presentación de propuestas educativas sobre estos, entre otros.

El curso Tecnologías de Aprendizaje Compartido y Distribuido se ofrece en modalidad 100\% en línea y tiene como propósito general analizar innovaciones tecnológicas y diseños educativos que ofrecen nuevas perspectivas de aprendizaje y de comunicación, y sus objetivos son: a) analizar procesos comunicativos vinculados con el uso de tecnologías de la comunicación, b) analizar la perspectiva tecnológica en la educación, c)analizar diferentes teorías sobre el aprendizaje colaborativo y distribuido, d) evaluar el proceso de diseño y desarrollo de un aprendizaje compartido y distribuido, y e) identificar las proyecciones de desarrollo tecnológico y educativo (UNED, 2010).

El curso Telecomunicaciones Educativas I se ofrece en modalidad $100 \%$ en línea y tiene como propósito general valorar diversas posibilidades que brindan las plataformas de comunicación e información al proceso educativo, como medios que favorecen el aprendizaje, la investigación y el aprendizaje multimedial y sus objetivos son: a) reconocer diferentes recursos que ofrece Internet para el apoyo del proceso educativo, b) valorar software y herramientas en línea, con base en guías previamente diseñadas, para la atención de estudiantes de 
diferentes niveles y c) diseñar propuestas con los diferentes recursos estudiados que ofrece la Red, con el propósito de apoyar el proceso de aprendizaje de los estudiantes en diferentes áreas del currículo (UNED, 2010).

La experiencia de pertenecer al proyecto OCW ha significado para la cátedra Tecnologías Informáticas para la Educación del Programa de Informática Educativa una oportunidad de dar a conocer sus cursos a nivel mundial mediante el sitio del proyecto, lo que conlleva a que tanto estudiantes como docentes de diferentes latitudes conozcan los planteamientos de estos cuya modalidad es 100\% en línea.

Es importante que otras cátedras que deseen comenzar con esta iniciativa y no tengan la experiencia necesaria cuenten con un ejemplo que se pueda replicar en el momento de desear incorporar sus cursos a esta modalidad.

Asimismo, significa un compromiso para los profesores diseñadores instruccionales y para el encargado de cátedra, que tienen a cargo cursos en el proyecto OCW la actualización constante de todos los materiales y recursos didácticos utilizados en éstos.

Por ser los cursos del área de Tecnología e Informática Educativa donde constantemente se implementan innovaciones tecnológicas y actualizaciones, es todavía mayor la responsabilidad de mantener una actualización permanente.

Por otro lado, el contar la cátedra con una representación en el proyecto OCW significa una gran oportunidad y un logro significativo, ya que esto permite valorar el hecho de que estos cursos pueden ser accedidos por pares académicos y por estudiantes, tanto a nivel nacional como internacional, que deseen aprender sobre el contenido de aquellos. También pueden ser consultados por usuarios que se encuentren en otros contextos, estén o no incorporados al proyecto OCW.

\section{Estadísticas de Visitas al Proyecto OCW de la UNED}

Desde la coordinación en la UNED del proyecto $\mathrm{OCW}$, se generan estadísticas, sobre información general, países desde donde se han accesado los cursos, fuentes desde donde se ha buscado el curso, entre otras informaciones, cada vez que se requiera.

De acuerdo con estadísticas enviadas regularmente por Hernández (2011) a los coordinadores de cursos del proyecto en la UNED, las cuales le facilitan a través de Google Analytics, consta que un período comprendido entre el 26 de enero del 2010 y el 7 de junio de 2011, los resultados generados con respecto a los cursos de la Cátedra Tecnologías de Aprendizaje Compartido y Distribuido y Telecomunicaciones Educativas I son los siguientes:

» Para la consulta de visitas al apartado denominado "Información de Contenido", se menciona que el curso Telecomunicaciones Educativas I se ha visitado 1291 veces a través de 25 países/ territorios. mientras que, para el curso Tecnologías de Aprendizaje Compartido y Distribuido, el mismo apartado se ha visitado 1029 veces a través de 25 pases/territorios (p. 1).

»Con respecto a la búsqueda denominada "Fuentes de entrada" de las visitas, esto es, de dónde provienen las visitas, el curso Telecomunicaciones Educativas I ha sido visitado 518 veces a través de 14 fuentes, entre estas fuentes se puede mencionar google, ocwconsortium.org, direct, search, uned.blackboard.com, uned.ac.cr, google.com.gt, google.co.cr, google.com, google.com. ec, Bing, google.co.ve, search.iminent. com, web.uned.ac.cr. Mientras que, para el curso Tecnologías de Aprendizaje Compartido y Distribuido, las visitas a la página del curso según la fuente han sido de 400 veces a través de las 
siguientes 13 fuentes: google, direct, ocwconsortium.org, google.com.co, search, bing, google.co.ve, google.com. $\mathrm{mx}$, uned.ac.cr, google.com.pe, search. iminent.com, google.com.ar, home. speedbit.com (p.1).

\section{CONCLUSIONES}

La incorporación de las instituciones de educación superior al proyecto Open Course Ware les permite tener un mayor posicionamiento a nivel mundial. El proyecto potencia en los docentes diseñadores instruccionales la búsqueda de la innovación y el incremento de iniciativas tendientes a fortalecer buenas prácticas educativas acompañadas por el uso de las tecnologías de la información y la comunicación.

Entre las ventajas más destacables para los estudiantes y profesores, se puede mencionar la accesibilidad en forma libre a través de la red de Internet a gran cantidad de contenidos, recursos, materiales didácticos y metodologías de trabajo provenientes de diversos planes de estudio de distintas universidades del mundo.

Lo anterior promueve la posibilidad de compartir iniciativas tendientes a fomentar la educación permanente y continua de quien accede a los cursos. Además, permite la socialización del conocimiento mediante una inmensa comunidad de aprendizaje como lo es el proyecto Open CourseWare.

Los académicos de educación superior tienen la oportunidad de utilizar, modificar y reutilizar diferentes materiales y recursos didácticos para el ejercicio de su práctica docente, que han sido ya validados por equipos interdisciplinarios expertos del área académica de las instituciones de educación superior.

Esto asegura estándares de calidad, dado que se puede ofertar a los usuarios recursos de procedencia confiable, todo esto dentro de un propósito: el mejoramiento de la docencia uni- versitaria y la formación permanente con apoyo de la tecnología.

Además de ofertar materiales y contenidos actualizados, según las tendencias mundiales en el contexto de las tecnologías aplicadas a la educación, bajo enfoque pedagógico y didácti$\mathrm{Co}$, realizado por todo un equipo interdisciplinario especializado en diferentes áreas, según los requerimientos de los cursos.

\section{BIBLIOGRAFÍA}

Charles, M. (2002). Mensaje del Rector. Recuperado el 1 de abril de 2010 de http://mit.ocw.universia.net/presidentspage.htm.

Graf \& Albright, (2007). Administración y Evaluación de la Tecnología Instruccional y la Educación a Distancia. Manual del Curso ITDE 8012. Nova Southeastern University.

Hernández, (2001). Informe Reunión Anual OCWC 2009 Monterrey, México. UNED 2009.

MIT-OCW, (2002). Unlocking Knowledge, Empowering Minds. Recuperado el 16 de marzo de http://ocw.mit.edu/OcwWeb/web/about/ about/index.htm.

OCW-UNED, (2011). Estadísticas del sitio OCW UNED. Material obtenido a partir de la herramienta Google Analytics.

OCW-Universia, (2007). Open Course Ware. Un Proyecto Estratégico para las Universidades. Recuperado el 15 de marzo de 2010 de www. universia.net.co/dmdocuments/universia_folleto_3.pdf.

OpenCourseWare, (2007). Un Proyecto Estratégico para las Universidades. Documento para la Difusión y Participación. Recuperado el 8 de marzo de http://www.universia.net.co/dmdocuments/universia_folleto_3.pdf.

Plasencia, D. (2006). El cielo como pizarra. Recuperado el 9 de marzo de 2010 de www. universia.edu.pe/noticias/principales/destacada. php?id=56761UNED, (2009). Misión. 
Recuperado el 5 de octubre del 2009 de http:// www.uned.ac.cr/rectori/mision.shtml

UNED, (2009). Visión. Recuperado el 5 de octubre del 2009 de http://www.uned.ac.cr/rectori/vision.shtml.

UNED, (2011). Programas de Bachillerato y Licenciatura en Informática Educativa.
Wiley, D. (2010). El contenido abierto es una publicidad potentísima para las universidades. Recuperado el 12 de marzo de 2009 de http:// www.universia.es/html_estatico/portada/actualidad/noticia_actualidad/param/noticia/ jbghh.html. 